(C)2019 IEEE

https://doi.org/10.1109/eem.2019.8916308

Madina, Carlos, Pirkko Kuusela, Marco Rossi, and Hamid Aghaie. "Optimised TSO-DSO Coordination to Integrate Renewables in Flexibility Markets." 20 19 16th International Conference on the European Energy Market (EEM) (September 2019). doi:10.110 9/eem.2019.8916308. 


\section{Optimised TSO-DSO Coordination to Integrate Renewables in Flexibility Markets}

\author{
Carlos Madina \\ Inés Gómez-Arriola \\ Sandra Riaño \\ TECNALIA \\ Derio, Spain \\ Marco Rossi \\ RSE \\ Milan, Italy
}

\author{
Pirkko Kuusela \\ VTT \\ Espoo, Finland \\ Hamid Aghaie
AIT
Vienna, Austria
}

\begin{abstract}
The necessary energy transition to decarbonize power systems is leading to increasingly important challenges for the operation of power systems. On the one hand, the intermittent nature of renewable generation requires system operators to procure ancillary services in larger volumes than in the past. On the other, the growing penetration of medium- and small-scale, flexible demand and storage systems in distribution networks could potentially offer network services, if they are aggregated effectively and there is an appropriate coordination between transmission system operators (TSOs), distribution system operators (DSOs) and aggregators. Therefore, an important topic to be analysed is whether distributed energy resources (DER) can replace traditional generation in the provision of ancillary services (AS), how this replacement will affect the system operators' roles and how to improve the coordination between TSOs and DSOs. This paper shows the results of the cost-benefit analysis (CBA) performed within the project SmartNet to assess the advantages or disadvantages of different TSO-DSO coordination schemes, as well as the follow-up activities to be carried out in the project CoordiNet.
\end{abstract}

Index Terms-CBA, TSO, DSO, Flexibility, Coordination.

\section{INTRODUCTION}

The replacement of fossil-fuel-based generation by renewable generation is leading to increasingly important challenges in terms of frequency stability, congestion management, voltage regulation and power quality, due to the variable behaviour of renewables. At the same time, there is a growing penetration of medium and small-scale, flexible demand and storage systems in distribution networks. These resources could provide network services if they are aggregated effectively and if there is an appropriate coordination between TSOs, DSOs and aggregators.

For this reason, it is interesting to analyse to which extent DERs can replace traditional generation in the services provision to network operators. The participation of these distributed resources in the AS markets will require a change in the roles of the distribution companies, as well as greater cooperation and coordination between them and the TSOs. The European Union itself recognized this need for updating roles (Article 32, [1]), by giving to the DSO responsibilities in terms of congestion management, enabling also the possibility of establishing dedicated market mechanisms to acquire the necessary flexibility (but not to balance the system frequency, whose management remains in the TSO's hand).

\section{FLEXIBILITY MARKETS SIMULATED}

Different TSO-DSO coordination schemes (CSs) and realtime market architectures were compared in the project SmartNet (http://smartnet-project.eu/) to find out which one could deliver the best compromise between costs and benefits for the system (similar work performed in [2], [3]):

1) Centralised AS market model (CS A): The TSO can contract AS directly with DER owners connected to the DSO grid. The DSO can procure and use DER to solve local grid issues, but not in this real-time centralised AS market.

2) Local AS market model (CS B): The DSO, via a local market, procures flexibility for solving local problems and, then, an aggregation of the remaining resources is transferred to the TSO, so that the TSO can contract DER only indirectly.

3) Shared balancing responsibility model (CS C): The TSO transfers balancing responsibility to the DSO. The DSO has to respect a pre-defined schedule at the TSO-DSO interface by using local DER (obtained via a local market) to fulfil its balancing responsibilities.

4) Common TSO-DSO AS market model (CS D): TSOs and DSOs contract DER in a common flexibility market to minimise total activation costs of flexibilities contracted. 
In order to compare which of these CSs provides a better compromise between costs and benefits, an ad-hoc platform was developed [4] to carry out simulations and evaluate the implementation of TSO-DSO interaction schemes, under plausible 2030 energy scenarios for three European regions (Northern Italy, Continental Denmark and the peninsular system of Spain), whose characteristics are reported below:

- Italy and Spain are expected to face a significant increase of photovoltaic (PV) and wind generation, which will be comparable to the total power capacity of conventional power plants. This renewable generation will be located mostly at distribution level.

- Thanks to the spread of electric vehicles, storage-based technologies are expected to have a significant potential in all the considered regions.

- Flexible thermal loads will shyly increase in all the scenarios, except in Denmark, where they will represent a significant portion of the available flexibility, as controllable heat pumps are expected to replace large combined heat and power (CHP) units by 2030 .

\section{SIMULATION PLATFORM}

The flexibility market considered in the SmartNet project, "Integrated Reserve Market", was aimed at solving real-time imbalances and congestions between gate closure of intraday markets and real time until the opening of the next intraday market session [5]. The market horizon can vary as a function of the market requirements, but in general it would last from 15 minutes to 1 hour.

The simulation environment was divided into three main layers, which are further detailed in [4], [6] and [7]:

1) The market layer integrates the market clearing algorithms, which process the bids proposed by the different market players and returns the optimal activations aimed at restoring the system balance and solving/avoiding network congestions.

2) In the bidding and dispatching layer, the bids that different agents (both traditional producers and retailers, as well as aggregators representing numerous flexible resources connected in distribution) send to the market layer are created. Market players use different algorithms to process the available flexibility of energy resources into bids and to translate market results into activations. More details about this process can be found in [8].

3) The physical layer simulates the physical processes of the electrical network (transmission and distribution) as well as of the generation, consumption and storage equipment connected to it. Hence, it simulates the effects of the activations on transmission and distribution networks, including the physics of each (flexible and non-flexible) device connected to them.

Three scenarios were created for each of the regions described above, representing three typical days, with different demand (wet appliances, thermostatically controlled loads and sheddable loads), solar irradiation and wind conditions.

\section{CBA}

A CBA was performed to identify the coordination scheme with the highest performance [9], [10]. With this purpose, the following metrics were selected and converted into monetary units:

1) Total mFRR cost: This indicator includes the total balancing cost of the market defined in SmartNet. The energy activated is remunerated at the nodal price resulting from the market clearing process. The mFRR activations are aimed to solve the network imbalance and to avoid congestions predicted in advance for the next time step.

2) Total aFRR cost: This is the cost of re-balancing the system after the mFRR market. In this case, an off-line aFRR market is simulated in order to optimally select the resources and the price for this reserve.

3) Unwanted measures (UM): Each coordination scheme requires a different market setup and a different level of detail for the grid model to be used in the market clearing process, which aims to solve and avoid congestion issues in the network. Some simplifications, required to allow for a faster execution of the market clearing algorithm, together with forecasting uncertainty, may create infeasibilities when dispatching the units cleared in the market. Hence, some bids accepted in the market clearing may create congestions that are not identified by the grid model used. In this case, grid operators must take emergency actions to re-dispatch some resources aiming to solve real congestions in the grid. Since it is assumed that re-dispatched resources are remunerated according to their actual cost for reaching the requested setpoint and the real unit cost are used to create the bids for the mFRR market, the UM are monetised at the mFRR bid price.

4) Information and communication technologies (ICT) costs: Since all investigated CSs feature very similar communication system requirements to monitor and operate DER, the cost estimation focused on the software development effort for the realization of aggregation and market optimization routines. It was assumed that Centralised AS market model (CS A) is a natural evolution of the current coordination and, hence, that it will be operational in 2030 . For this reason, the investigation evaluates costs to upgrade from CS A to alternative schemes by calculating IT system development and upgrade costs. No indirect costs, e.g. increase of grid monitoring, are considered.

In addition to these metrics, the $\mathrm{CO}_{2}$ emissions were also calculated as a complementary metric for informative purposes. Since the cost of the $\mathrm{CO}_{2}$ emissions was already included in the bids sent by the aggregators to the mFRR market, this metric was not monetised.

For each of the simulated typical days, the total cost of each CS is the addition of the four metrics previously mentioned. Then, the total annual costs are obtained by estimating the number of occurrences of each typical day, based on the real conditions in 2018 . 


\section{RESULTS \& DISCUSSION}

Next figures show the results obtained for each country in the four coordination schemes considered.

As Figure 1 shows, CS A is the cheapest CS in Denmark and $\mathrm{CS} C$ the most expensive one. The amount of mFRR energy managed in each CS (which is definitively larger that the aFRR one) is the cost driver in the simulated scenario. Since the scenario considered infrequent distribution congestions, the results for CS A, CS B and CS D are very similar, and the main difference is caused by the ICT costs.

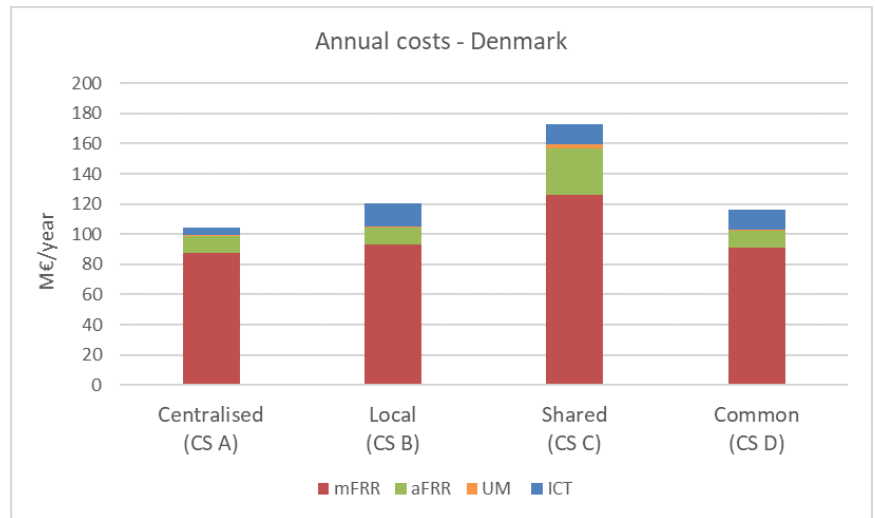

Figure 1: Annual costs in the Danish scenario

mFRR costs, which are very similar for all CSs, represent also the main CBA component in Italy, as shown in Figure 2. The main difference between the CSs is determined by the aFRR cost. In the scenarios considered, the most efficient CSs are the CS B and CS D, although the costs for all CSs are very similar. The large activated volumes of frequency restoration reserves make the UM and ICT costs practically negligible.

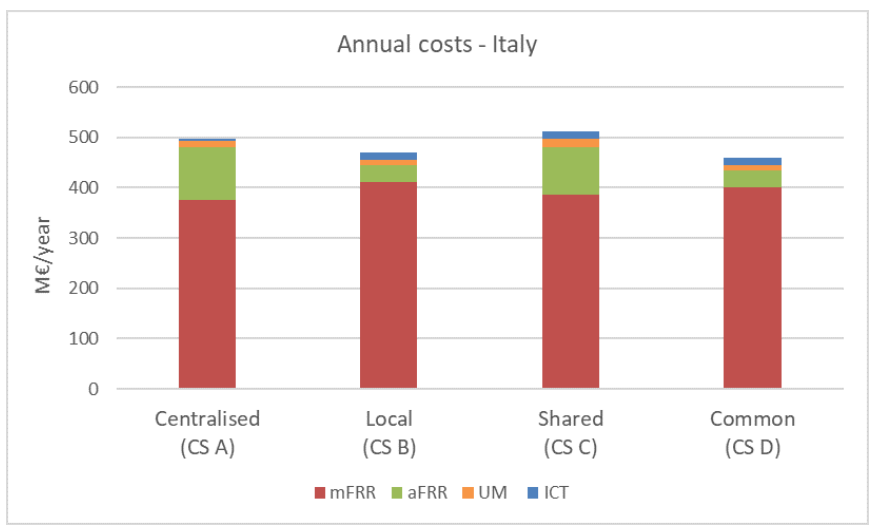

Figure 2: Annual costs in the Italian scenario

In the Spanish case, Figure 3, the CBA results of CS A, CS B and CS D are very similar, while the CS C results to be the most expensive. The impact of ICT and UM is negligible with respect to the rest of costs. Being the total costs of CS A, CS B and CS D comparable and the mFRR costs very similar in all CSs, the difference between these CSs is determined by the weight that the terms aFRR, UM and ICT have on the total amount. The inefficiency of CS C is determined by the aFRR contribution.

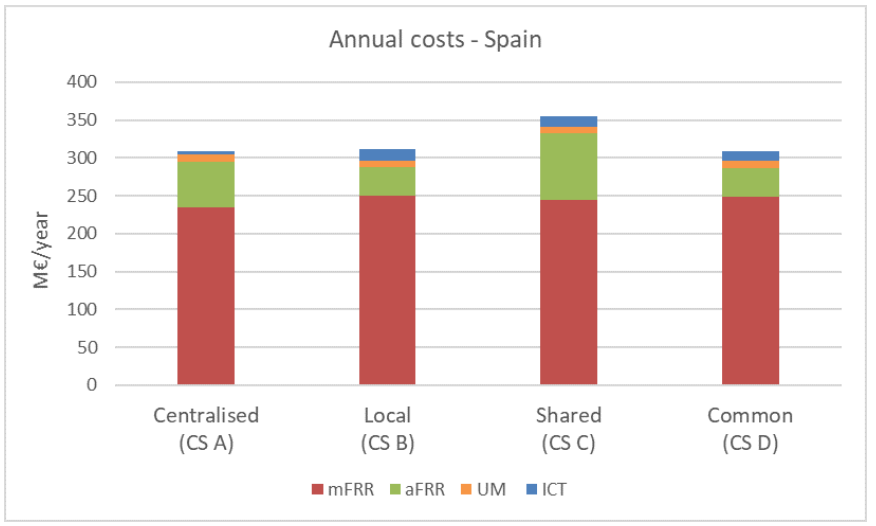

Figure 3: Annual costs in the Spanish scenario

The $\mathrm{CO}_{2}$ emissions have not been monetized, since the bids sent by aggregators already include a cost for the $\mathrm{CO}_{2}$ ton, but they have been calculated and reported in Figure 4 (note that the scale is adapted for an easier comparison).

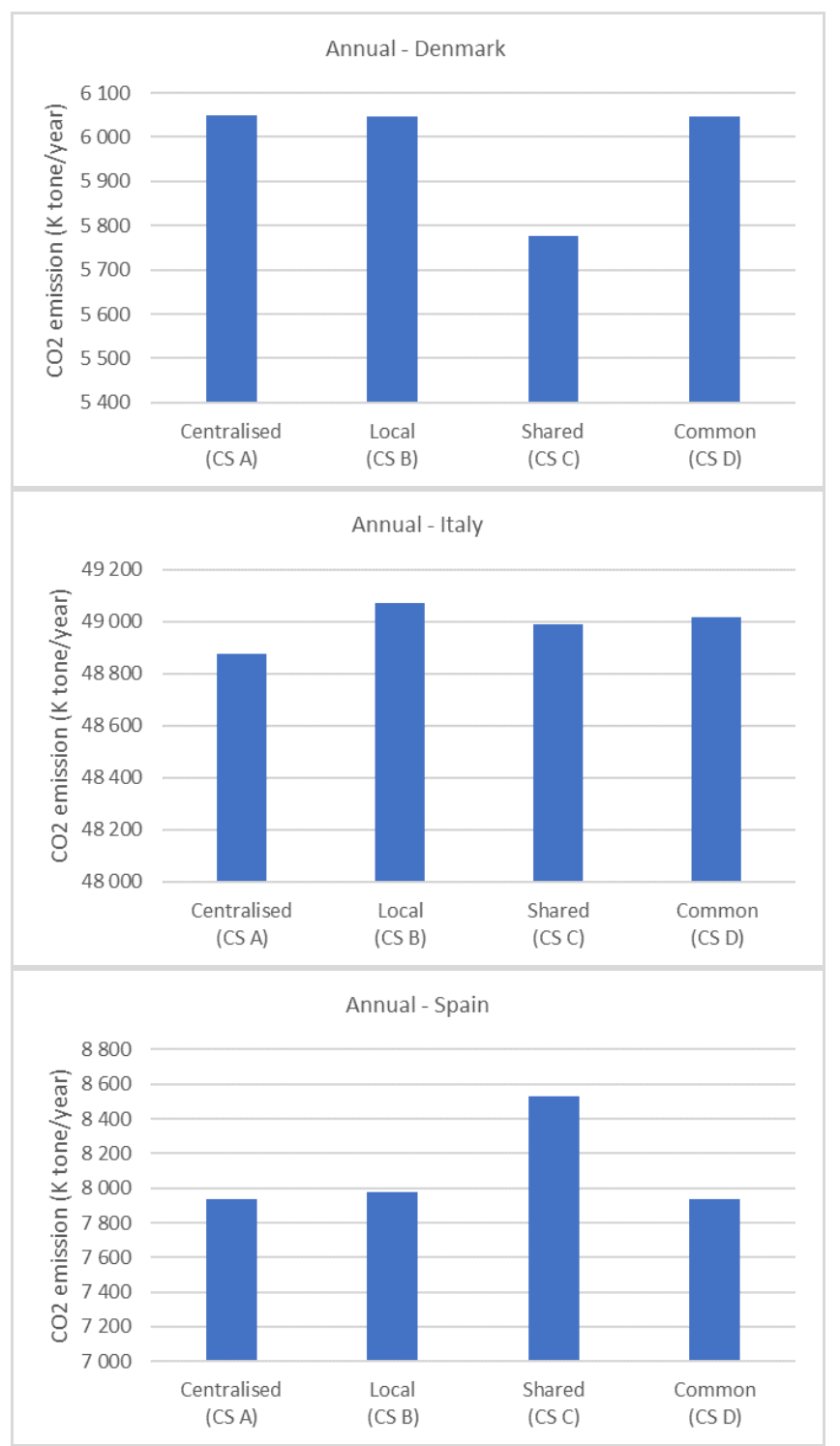

Figure 4: Annual $\mathrm{CO}_{2}$ emissions in the three national scenarios 
The effectiveness of the TSO-DSO coordination schemes depends on the level of services requested by the DSO:

- In case of few congestions at distribution level (forecasting errors are comparable to the possibility of having congestions in distribution grid), CS A (centralized CS) has higher economic performance than other options. This is the case of Denmark, where the contribution of wind power (located on transmission network) is higher than the contribution from PV and, thus, forecasting errors are expected to be higher, while the congestions at distribution level are likely to be less frequent.

- When distribution congestions are significant (and predictable), the adoption of common (CS D) or local markets (CS B) results to be beneficial. This is the case in Italy, where generation located in distribution grids, such as PV, contributes more to the electricity mix and, hence, congestions are expected to happen more frequently.

- In any case, the most relevant cost component is mFRR in all the cases, while UM and ICT costs account for only a small share.

The implementation of two-step markets (CS B and CS C) is generally less efficient than optimising in a single step:

- Regarding CS B, the results are similar to the ones returned by CS D, although slightly more expensive in the scenarios simulated. This effect was quite expected since CS B optimises in two separated steps and sub-optimum solutions are likely to be obtained.

- The shared balancing responsibility model (CS C) is clearly the least efficient CS in all the countries. In addition to optimising in two steps, it introduces an additional constraint by fixing the active power exchange at the TSO-DSO interconnection, which further takes the solution away from the absolute optimum obtained in CS D.

- Both CS B and CS C may suffer from scarcity and/or illiquidity of resources, which would further decrease their efficiency.

- Yet, in rare circumstances (i.e. severe congestions at transmission level) two-step market architectures can be more beneficial than other schemes, as market separation may prevent the spreading of high nodal prices among distribution and transmission systems.

Under the scope restrictions and the formulation of the ICT cost estimation problem, the main finding was that ICT costs are much lower than the operational costs in the different market arrangements and that their impact in the scoring of CSs is almost negligible (and subject to uncertainties). The aggregators will bear a large portion of these ICT costs (communications with DERs, aggregation software, etc.) and the issue of the last kilometre DER communications must be solved. However, this does not affect the scoring of the CSs, since a similar communication cost is expected for all CSs.

Some country-specific deductions can also be extracted:
- In Italy, where congestions at distribution level are noticeable, the upgrade from CS A to CS B/D is convenient and not jeopardized by ICT costs.

- In Spain, with average congestions at distribution level, ICT costs are comparable to the benefits of adopting CS B/D rather than maintaining CS A.

- In Denmark, with low congestions at distribution level and high forecasting errors, the implementation of all CSs with DSO services failed.

The $\mathrm{CO}_{2}$ emissions in Italy are one order of magnitude higher than in the other two countries, because each country has a different energy mix in the scenarios considered. However, all CSs feature similar $\mathrm{CO}_{2}$ emissions with less than $7 \%$ of difference. The main reason is that mFRR activations are small if compared to the total energy demand (and even more the aFRR and UM activations), so the efficiency in TSO-DSO coordination does not have a big impact in the overall $\mathrm{CO}_{2}$ emissions. In the specific case of $\mathrm{CS} \mathrm{C}$, the suboptimal mFRR activations drive to more visible impacts on $\mathrm{CO}_{2}$, except when forecasting errors are comparable to the probability of having congestions in distribution (Denmark), as $\mathrm{CS} \mathrm{C}$ becomes more efficient in terms of $\mathrm{CO}_{2}$ emission too.

\section{CONCLUSIONS}

In view of the outcomes obtained during the project, and considering the scenarios analysed within it, the adoption of CS C results to be the least efficient CS in all the countries and, therefore, technical reasons could advise to continue centralizing balancing responsibility to TSOs. However, depending on the impact of the congestions at distribution level, the congestion management responsibility could be shared between TSOs and DSOs:

- Traditional TSO-centric schemes could stay optimal if distribution networks do not show significant congestions, which is likely in the very near-future scenarios where the distribution grid planning continues affected by the fit-and-forget reinforcement policy. However, this regulatory trend may soon be modified, so that the DSOs' remuneration will give more importance to their investments in intelligence (OPEX) rather than in grid elements (CAPEX). Then, a more advanced coordination between TSO-DSO, like the proposed CS B or CS D, should be deployed for a more efficient management of the system.

- More advanced centralized schemes incorporating distribution constraints (CS D) show higher economic performances, but their performance could be undermined by big forecasting errors. Hence, it is of paramount importance to improve the forecasting techniques, to increase the market clearing frequency and to shift the gate closure as much as possible towards real-time.

- The two-step optimization process implies that the decentralized schemes are less efficient than singlestep processes. Scarcity of liquidity and potential impact of local market power (not investigated in SmartNet), along with extra constraints introduced to 
avoid counteracting actions between local congestion market and balancing market (e.g. increasing the system imbalance while solving local congestions), have an additional negative impact in the economic efficiency of decentralized schemes. The effect is especially important in CSC, where added constraints (e. g. imposing flow at the TSO-DSO interface in CS C) further decrease the CS efficiency.

- The local congestion markets should have a "reasonable" size and guarantee a free competition by a sufficient number of actors, in order to prevent scarcity of liquidity and the power exercise by the local markets. This may require that small DSOs pool-up in order to ensure the required market size.

- $\quad$ CS D has apparent advantages over the rest of CSs in the more than likely mid-term scenario in which the fit-and-forget reinforcement remuneration approach is abandoned and the forecasting errors are more accurately calculated. Yet, the network observability cannot be pushed till single low-voltage nodes due to complexity reasons. As a result, the observability level to be deployed for each specific case and country must be determined, taking into account that increasing the observability of distribution grids implies new important investments by the system.

- Once the most advisable CS at system-level is selected, a business-level analysis is needed to assess the economic impact of the different CSs for all the relevant actors in order to guarantee that all the involved participants have a profitable business case. The appropriate allocation of costs and benefits among the actors is of utmost importance when selecting the CS, since this issue may strengthen or threaten the deployment of the proposed CS.

\section{FOLLOW-UP: COORDINET PROJECT}

The work performed in SmartNet is being continued in the project CoordiNet (https://coordinet-project.eu/). CoordiNet aims to demonstrate how DSOs and TSOs shall coordinate to procure grid services in the most reliable and efficient way through the implementation of large scale "TSO-DSOConsumer" demonstration projects.

It is intended to demonstrate how the TSO (responsible for ensuring the balance of the power system in its control area and managing the very high voltage transmission grid) and the DSOs (managers of the high-, medium- and low-voltage distribution grids), can act in a coordinated way, to purchase and activate grid services, promote the cooperation of all actors and remove the barriers for customers to have an active market participation. In that sense, three large-scale demo projects are being implemented in Spain, Sweden, Greece, consisting of ten demonstration pilots (four in Spain, four in Sweden and two in Greece) and representing various boundary grid, climatic, load and generation conditions. For that purpose, one of its main aims is to define or adapt, demonstrate and promote future standardized grid services and related market platforms to enable a seamless pan-European electricity market.
As a first step, the flexibility requirements by TSOs and DSOs have been analysed. From this analysis, some flexibility products and services have been identified. These products and services are expected to be used across Europe and, thus, they will be demonstrated in the different pilots. Moreover, the coordination schemes defined in SmartNet have been adapted to further clarify the roles of both TSOs and DSOs.

With all this information, each demonstration pilot has defined the business use cases to be deployed at each site. Those business use cases will be the basis for the economic assessment to be performed within CoordiNet. The impact of the use of new products and services with the refined coordination schemes will be evaluated in the three demo countries, in order to determine the advantages for the overall power system. This analysis is in line with the one performed in SmartNet, but the scope is extended.

Then, the business use cases defined by the demonstration pilots will be assessed, to see under which conditions all the stakeholders in the value chain can have a positive outcome, that is whether the costs and benefits of deploying these coordination schemes, products and services are properly allocated among them.

\section{ACKNOWLEDGEMENT}

These projects have received funding from the European Union's Horizon 2020 research and innovation programme under grant agreements No 691405 \& No 824414.

\section{REFERENCES}

[1] European Commission, "Proposal for a Directive of the European Parliament and of the Council on common rules for the internal market in electricity", COM (2016) 864 final/2, Brussels.

[2] S. W. Kim, M. G. Pollit, Y. G. Jin, J. Kim, 2018, “A contractual framework for sharing balancing responsibility between transmission system operator and distribution system operators"

[3] S. Burger, J. Jenkins, C. Battle, I. Pérez-Arriaga, 2018, "Restructuring revisited: Competition and Coordination in Electricity Distribution Systems", MITCEEPR (No. CEEPR WP 2018-007).

[4] M. Rossi, G. Viganò, G. Migliavacca, Y. Vardanyan, R. Ebrahimi, G. Leclercq, P. Sels, M. Pavesi, T. Gueuning, J. Jimeno, N. Ruiz, G. Howorth, J. Camargo, C. Hermans, F. Spiessen, H. Svendsen, 2019, "Testing TSO-DSO Interaction Schemes for the Participation of Distribution Energy Resources in the Balancing Market: the SmartNet Simulator", CIRED 2019, paper n ${ }^{\circ} 1158$.

[5] G. Migliavacca, M. Rossi, D. Six, M. Dzamarija, S. Horsmanheimo, C. Madina, I. Kockar and J. M. Morales, 2017, "SmartNet: H2020 project analyzing TSO-DSO interaction to enable ancillary services provision from distribution networks", CIRED - Open Access Proceedings Journal, vol. 2017, no. 1, 1998-2002. DOI 10.1049/oapcired.2017.0104.

[6] G. Viganò, M. Rossi, P. Sels, G. Leclercq, T. Gueuning, M. Pavesi, Y. Vardanyan, R. Ebrahimy, J. Jimeno, N. Ruiz, G. Howorth, J. Camargo, C. Hermans, F. Spiessen, H. Svendsen, 2019, "D4.1. SmartNet simulation platform". SmartNet project deliverable. Available on-line at: http://smartnet-project.eu/wp-content/uploads/2019/06/D4.1.pdf

[7] H. Svendsen, M. Rossi, G. Viganò, J. Merino, J. Le Baut, H. Sawsan, 2019, "D4.2. Scenario setup and simulation results" SmartNet project deliverable. Available on-line at: http://smartnet-project.eu/wpcontent/uploads/2019/07/D4.2 20190628 V1.0..pdf

[8] J. Jimeno, N. Ruiz, C. Madina, 2019, “Aggregation of Thermostatically Controlled Loads for Flexibility Markets”, CIRED 2019, paper nº 1502.

[9] C. Madina, S. Riaño, I. Gómez, P.Kuusela, H. Aghaie, J. Jimeno, N. Ruiz, M. Rossi, G. Migliavacca, 2019, “Cost-Benefit Analysis of TSO- 
DSO coordination to operate flexibility markets", CIRED 2019, paper $\mathrm{n}^{\circ} 1632$.

[10] I. Gómez, S. Riaño, C. Madina, M. Rossi, P. Kuusela, P. Koponen, H. Aghaie, G. Migliavacca, E. Rivero, H. Xu, I. Kockar, 2019, "D4.3.
Cost-benefit analysis of the selected national cases" SmartNet project deliverable. Available on-line at: http://smartnet-project.eu/wpcontent/uploads/2019/06/D4.3.pdf 